\title{
Falta de Competencia y Sobrecarga Laboral como Estresores en Prácticas Clínicas Intrahospitalarias
}

\author{
Lack of Competence and Work Overload as Stressors \\ in Intrahospital Clinical Practices
}

\author{
Edith Morales Herrera; ${ }^{1}$ Claudia Amigo Rojas; ${ }^{1}$ Miguel Bustamante Ubilla; ${ }^{2}$ \\ 1 Facultad de Ciencias de la Salud, Universidad de Talca \\ 2 Facultad de Economía y Negocios, Universidad de Talca
}

\section{Resumen}

Este trabajo estudia la falta de competencia y sobrecarga laboral como estresores en prácticas clínicas intrahospitalarias de enfermería. Se aplicó una metodología cuantitativa por medio del cuestionario bilingüe Kezkak, el cual considera nueve dimensiones de estrés. Se realizó un piloteo del instrumento con docentes para verificar entendimiento y comprensión del mismo. Los estudiantes fueron contactados vía on-line lográndose una cobertura de $89,6 \%$. Se pudo concluir que los estudiantes de enfermería presentaron estrés durante las prácticas clínicas intrahospitalarias preferentemente al inicio. Dentro de los principales factores de estrés destacan la falta de competencia y sobrecarga de trabajo de los estudiantes de enfermería en sus periodos de prácticas clínicas en los servicios de Medicina y Cirugía.

Palabras clave: Prácticas clínicas; estudiantes de enfermería; estresores; estrés

\section{Abstract}

This work studies the lack of competence and work overload as stressors in nursing hospital clinical practices. A quantitative methodology was applied using the Kezkak bilingual questionnaire, which considers nine dimensions of stress. The instrument was piloted with teachers to verify the realization and understanding of the instrument. The students were contacted via on-line achieving a coverage of $89.6 \%$. It could be concluded that the nursing students presented stress during in-hospital clinical practices, preferably at the beginning. Among the main stress factors, the lack of competition and work overload of nursing students in their periods of clinical practices in the Medicine and Surgery services stand out.

Keywords: Clinical practices; nursing students; stressors; stress 


\section{INTRODUCCIÓN}

Las prácticas clínicas son un elemento fundamental en la formación de los estudiantes de enfermería, ya que les permiten aplicar los conocimientos teóricos y desarrollar habilidades para la prestación de cuidados a los pacientes López y Sánchez (2005). Según destaca Moya, Larrosa López, López, Morales y Gómez (2013). Permitiéndoles comparar estos conocimientos aprendidos en las aulas con los de la práctica habitual de los centros asistenciales al realizar sus prácticas clínicas (López \& López, 2011).

Sin embargo, estas prácticas clínicas provocan estrés en los estudiantes de enfermería según refiere, Aravena (2017), quien realizó un estudio que evidenció que estas prácticas generan situaciones de estrés, por el contacto de los estudiantes con el sufrimiento, la muerte y las necesidades humanas. En este mismo ámbito Mahat (1996), estudió los estresores que afectan al ámbito clínico, observando que el 50\% de los casos tenían relación con el área interpersonal (López et al., 2011).

Para medir el estrés causado por las prácticas clínicas se utilizó el cuestionario Kezkak (Gac Sanit 2003), el cual ha sido validado tanto a nivel internacional como nacional en diferentes áreas de estudio, incluyendo el área de docencia.

Desde la perspectiva de las implicaciones laborales de una práctica clínica (Da Silva, 2006), se identifican diversos determinantes críticos del estrés (Celis, et al., 2001), los cuales son relevantes por sí mismos pero que a su vez, se potencian al actuar en conjunto debido a la constante competencia y relaciones internas que pueden terminar siendo preocupantes al interior de las organizaciones, especialmente las clínicas dada su complejidad (Davis y Newstrom, 1993), sobretodo en áreas en donde trabajadores y/o funcionarios (Gonzáles, 2017), deben responder a las necesidades del servicio que ofrecen (Forrellat, 2014), relacionado directamente con la calidad de los servicios sanitarios.

En estudios realizados con profesionales de enfermería, se ve que algunas fuentes de estrés estudiadas están presentes a lo largo de la vida profesional, como, "contacto con el sufrimiento", "impotencia e incertidumbre", "implicación emocional", "no controlar la relación con el enfermo", "resultar dañado en la relación con el enfermo", "relación con compañeros” y "sobrecarga laboral” (Zupiria-Gorostidi, 2006).

Otros estudios destacan que la falta de competencia, impotencia e incertidumbre, contacto con el sufrimiento y riesgo de dañarse en la relación con el enfermo (Cobo, Carbonell, Rodríguez, Vivo, Castellanos \& Sánchez, 2010), estimulan una percepción elevada de estrés durante las prácticas clínicas, mismas que, según López et al (2011), permiten a los alumnos comparar conocimientos teóricos adquiridos con los de la práctica habitual de los centros asistenciales, desarrollar habilidades y actitudes propias de los profesionales sanitarios, que los habilitan para enfrentarse a un nuevo rol para el que aún no se está completamente preparado y una de cuyas soluciones sería incrementar la duración de la carrera asociada al desarrollo de especialidades en enfermería (Pérez, Alameda \& Albéniz, 2002).

Complementariamente, estudios realizados con profesionales de enfermería (Gonzáles, 2017), confirman que la práctica clínica incide sobre la calidad de vida laboral activando el estrés haciéndose evidente en su desempeño y, a la larga, en la vida profesional (Granados, 2011). Estas evidencias son claramente apreciables en el sector de la salud cuando el practicante está en "contacto con el sufrimiento", debe superar sus propios sentimientos de "impotencia e incertidumbre" para avanzar hacia estados de control de los efectos e "implicación emocional” que le produce su desempeño laboral y que materializa en la denominada "sobrecarga laboral” (Zupiria-Gorostidi, 2006).

Analizados los argumentos teóricos anteriores, el presente estudio determina las principales causas de estrés de los estudiantes de tercer año de la carrera de enfermería de una Universidad pública, en relación a las prácticas clínicas realizadas en el área intrahospitalaria. Para ello se identificaron las causas más prevalentes de la falta de competencia y sobrecarga que presentan los estudiantes de enfermería durante las prácticas clínicas intrahospitalarias. 


\section{MÉTODO}

El estudio fue no experimental adoptando el enfoque cuantitativo, correlacional y de asociación de estresores laborales para analizar la evolución de las variables debidamente operacionalizadas. El instrumento utilizado fue el cuestionario bilingüe de estresores de los estudiantes de enfermería en las prácticas clínicas, Kezkak, creado por Zupiria Gorostidi; Uranga Iturriotz; Alberdi Erize \& Barandiaran Lasa, del Departamento Enfermería II; Escuela Universitaria de Enfermería de Donostia-San Sebastián, Universidad del País Vasco, San Sebastián, España (Zupiria, et al. 2003).

El instrumento midió los estresores a partir de 50 ítems que reflejan distintas situaciones potencialmente estresantes a las que pueden enfrentarse los estudiantes en el transcurso de sus prácticas clínicas (Tabla 1). Para cada ítem se establecen cuatro (4) respuestas en una escala tipo Likert (1932) donde (0) = nada; (1) = algo, (2) = bastante y (3) = mucho. Se consideró estrés a todos aquellos ítems que obtuvieron una puntuación media superior a 1,5 .

Tabla 1. Descripción de reactivos independientes o ámbitos de estrés

\begin{tabular}{ll}
\hline Falta de competencia & $\begin{array}{l}\text { Se entiende como no tener la capacidad de hacer bien el trabajo y perjudi- } \\
\text { car al paciente y posiblemente al que da la atención, (preguntas } \mathrm{N}^{\circ} 15,16, \\
\\
13,4,2,6,26,1,3,17,5) .\end{array}$ \\
\hline Sobrecarga & $\begin{array}{l}\text { Entendido como exceso de trabajo y de horario de prácticas, (preguntas } \mathrm{N}^{\circ} \\
35,36,34,30,31) .\end{array}$
\end{tabular}

El cuestionario recoge además datos demográficos tales como edad, sexo, procedencia, estudios previos de pre grado y situación económica (Eyssautier, 2006), además de antecedentes académicos como si ha reprobado alguna asignatura en su carrera, posesión de alguna beca relacionada con su desempeño académico. En lo sustancial, recoge la percepción de los estudiantes respecto de algún problema del ánimo, sentimiento de estrés, a que atribuye sus percepciones, si ha si ha tenido algún problema con algún paciente, que lo identifique y que finalmente lo explique brevemente.

La muestra estuvo constituida por un censo (Eyssautier, 2006), realizado a los estudiantes de la carrera de enfermería que cursaban tercer año y que realizaron prácticas clínicas intrahospitalarias de 20 días en el servicio de medicina y cirugía durante el semestre primavera 2016, con edades que fluctuaron entre 18 y 27 años y más, siendo un $73,9 \%$ de sexo femenino y un $26,1 \%$ de sexo masculino.

Los estresores laborales fueron identificados entre las situaciones relacionadas con el trabajo asignados a los estudiantes en sus prácticas profesionales, considerando que éstas puede provocar una respuesta de estrés en la persona evaluada pero sin modificar conductas o alterar el entorno en el cual se desarrollaron Cobo et al. (2010). Refieren que el entorno sanitario otorga numerosos estímulos generadores de estrés, debido al contacto con las enfermedades, el dolor, el sufrimiento, la muerte de los pacientes, o por tener que desempeñar un nuevo rol para el que aún no se está completamente preparado, corroborado por Antolín, Purialto, Moure y Quinteiro (2007).

El software estadístico a utilizar para el análisis de datos fue el sistema informático Statistical Package for the Social Sciences (SPSS) 15.

\section{RESULTADOS}

Los resultados se presentan en dos partes, una primera que describe las características de la muestra (Tabla 2) y una segunda que analiza los factores que provocan estrés laboral (Tabla 3). 
Tabla 2. Información demográfica y de antecedentes estudiantiles

\begin{tabular}{|c|c|c|c|}
\hline & n & $\%$ & $\%$ acumulado \\
\hline \multicolumn{4}{|l|}{ Edad } \\
\hline 18 a 20 años & 9 & 13.4 & 13.4 \\
\hline 21 a 23 años & 50 & 74.6 & 88 \\
\hline 24 a 26 años & 5 & 7.5 & 95.5 \\
\hline 27 años o más & 3 & 4.5 & 100 \\
\hline \multicolumn{4}{|l|}{ Sexo } \\
\hline Hombre & 26.1 & 26.1 & 26.1 \\
\hline Mujer & 73.9 & 100 & 100 \\
\hline \multicolumn{4}{|l|}{ Estudios previos de pre grado } \\
\hline Sí & 4 & 5.8 & 5.8 \\
\hline No & 65 & 94.2 & 100 \\
\hline \multicolumn{4}{|c|}{ Estudios de pre-grado previos realizados } \\
\hline Fonoaudiología & 2 & 50 & 50 \\
\hline Tecnología médica & 1 & 25 & 75 \\
\hline Licenciatura en ciencias & 1 & 25 & 100 \\
\hline \multicolumn{4}{|c|}{ Ha reprobado alguna asignatura en su carrera } \\
\hline Sí & 32 & 46.4 & 46.4 \\
\hline No & 37 & 53.6 & 100 \\
\hline \multicolumn{4}{|c|}{ Califique su situación económica (según su percepción) } \\
\hline Mala & 1 & 1.4 & 1.4 \\
\hline Regular & 48 & 69.6 & 71 \\
\hline Buena & 18 & 26.1 & 97.1 \\
\hline Excelente & 2 & 2.9 & 100 \\
\hline \multicolumn{4}{|c|}{ Usted tiene alguna beca relacionada con su desempeño académico } \\
\hline Sí & 36 & 52.2 & 52.2 \\
\hline No & 33 & 47.8 & 100 \\
\hline
\end{tabular}

El análisis descriptivo muestra los datos demográficos y académicos de los estudiantes analizados. En general, el 74,6\% de los estudiantes de enfermería estuvieron en el rango de edad de 21 a 23 años y un 73,9 \% eran mujeres, además, el 94,2\% no tenían estudios previos de pregrado.

De los 4 estudiantes que presentaron estudios previos de pregrado, dos estudiaron Fonoaudiología, uno Tecnología Médica y uno Licenciatura en Ciencias. Por otra parte, el 53,6\% de los estudiantes de enfermería de tercer año que realizaron prácticas clínicas intrahospitalarias no reprobaron ninguna asignatura de su carrera.

En lo económico, el 69,6\% de los estudiantes calificaron su situación económica como "regular", y que un 52,2\% mantenía alguna beca relacionada con su desempeño académico.

\section{Factores que provocan estrás laboral}

Se analizaron los factores del instrumento de acuerdo con el número de reactivos asociados y que se describen en la Tabla 3.

En general y analizando las frecuencias porcentuales más altas obtenidas del trabajo de campo, se aprecia que a un 84,1\% de los estudiantes de enfermería de tercer año, les preocupa mucho y bastante, por supuesto en términos bastante coloquiales en el contexto estudiantil, "Meter la pata "durante las prácticas clínicas intrahospitalarias.

Datos complementarios equivalentes a un $75,3 \%$, permiten determinar que a una alta proporción de los estudiantes de enfermería les preocupa bastante y mucho "Recibir órdenes contradictorias", las cuales les agobian y por supuesto les estresan, precisamente por la alta carga laboral que enfrentan en las prácticas clínicas. 
Tabla 3. Frecuencia absoluta y relativa de estresores de la práctica clínica intrahospitalaria

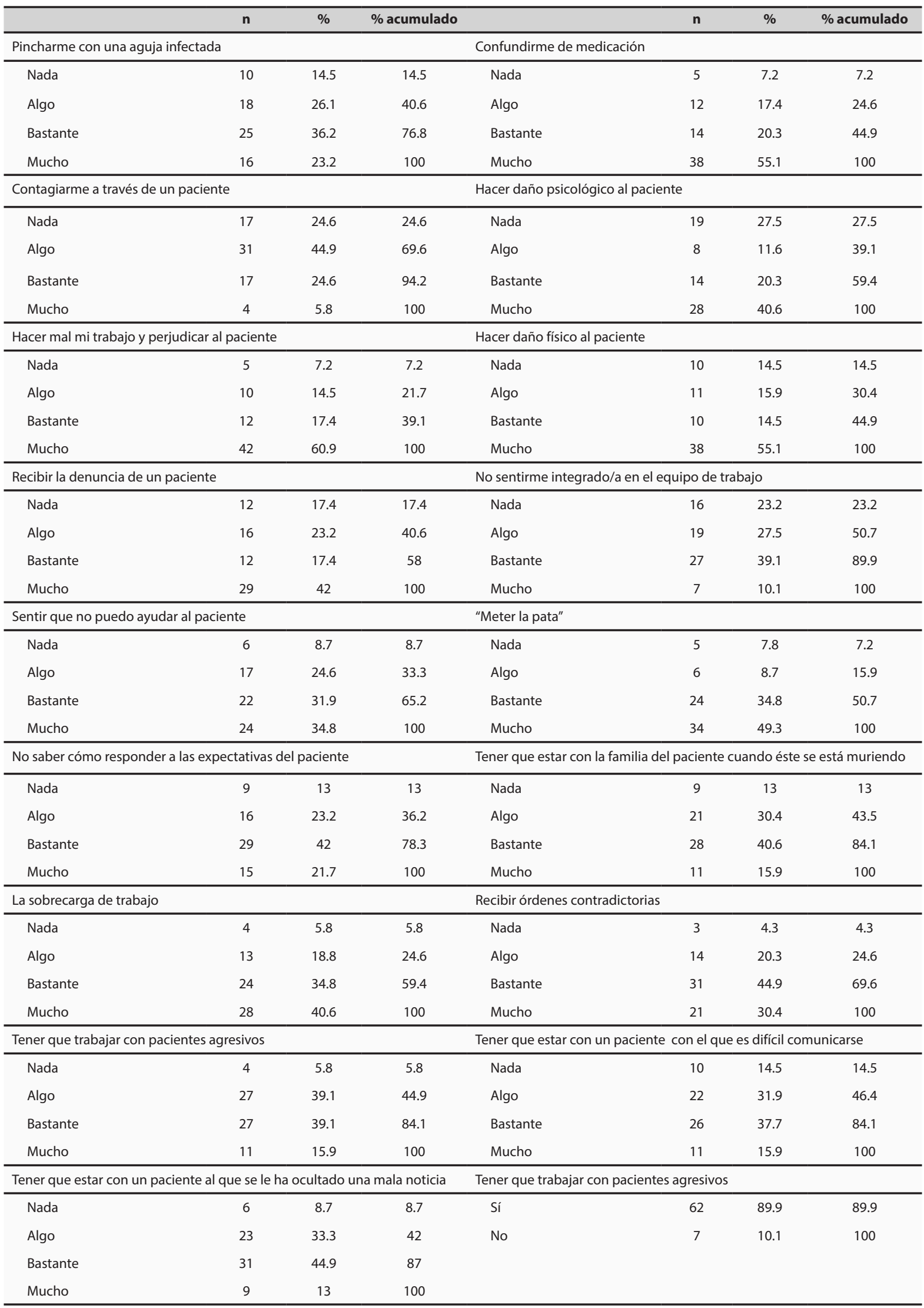


Por último, en forma general y agregada respecto de las exigencias de carga y sobre carga de trabajo, denominada en la entrevista "sobrecarga que presentan los estudiantes de enfermería en el transcurso de su práctica clínica intrahospitalaria”, se pudo determinar una alta frecuencia de casos, dónde se logró calcular que que a un relevante $75,4 \%$ de los practicantes de enfermería de tercer año, les preocupa mucho y bastante la "sobrecarga de trabajo" durante sus respectivas actividades intrahospitalarias que les fueron asignadas.

\section{DISCUSIONES}

Contrastando los hallazgos determinados en esta investigación con estudios relacionados pertinentes y consistentes con lo que aquí se analiza, en general se pueden identificar ciertas regularidades.

Respecto de las causas más prevalentes de la falta de competencia, destaca el hecho que a un 78,3\% de los estudiantes de enfermería de tercer año, les preocupa mucho y bastante "Hacer mal mi trabajo y perjudicar al paciente". Lo que corrobora, de Dios, Varela, Braschi y Sánchez (2017), ya que refieren que es necesario tener conocimientos sólidos y habilidades técnicas, las cuales se adquieren con la experiencia adquirida a través del ejercicio de la profesión, corroborado por Cobo, et al. (2010) y revalidado por López y López (2011).

En general, al 75,4\% de los estudiantes les preocupa mucho y bastante "Confundirse de medicación" y al 69,6\% de los estudiantes les preocupa mucho y bastante "Hacer daño físico al paciente”, durante las prácticas clínicas intrahospitalarias. Corroborado por Pérez, Alameda y Albéniz, (2002), quienes destacan el miedo como principales protagonistas de su práctica, lo que favorece cualquier daño o equivocación al atender al paciente. Además, Moya et al. (2003), reafirman esto al considerar que las situaciones de estrés que viven con mayor intensidad se deben a la inseguridad que representa el enfrentarse a situaciones nuevas y al miedo a no saber responder.

Destaca así mismo que a un 84,1\% de los estudiantes les preocupa mucho y bastante "Meter la pata "durante las prácticas clínicas intrahospitalarias. Esta percepción, ha sido ratificada por Velásquez, Cornejo, Bustamante y Mella (2018), los cuales concluyeron que para el manejo de este estresor en particular es "el desarrollo de habilidades sociales, lo cual es fundamental para poder realizar con éxito el trabajo, mientras se adquieren a lo largo de los años, conocimientos, destrezas y seguridad en sí mismos”, corroborado por López, Orbañanos y Cibanal (2013).

En relación con la sobrecarga que presentan los estudiantes de enfermería y que llega a un 75,4\% a quienes les preocupa mucho y bastante la "sobrecarga de trabajo", se pudo verificar que estos resultados son consistentes con los resultados obtenidos por McGrath, Boore (2003), consignando que la sobrecarga laboral es una fuente de estrés importante. Confirmando esto Greenglass, Burke y Fiksenbaum (2001), quienes aseguran que la gran sobrecarga laboral es la principal fuente de estrés de este colectivo.

Adicionalmente, a un 75,3\% de los estudiantes de enfermería les preocupa bastante y mucho "Recibir órdenes contradictorias", apreciación que ha sido corroborada por Moya et al (2013), quienes aseguran que los estudiantes no se han enfrentado a estas situaciones y que solo tienen miedo a no ser capaces de resolver correctamente a las situaciones que puedan verse enfrentados, reafirmado por Antolin, et al. (2007).

Finalmente al solicitarle a los estudiantes que realizaran una autopercepción de estrés, la Tabla 3 muestra que un 89,9\% de los estudiantes se sintió estresado durante sus prácticas clínicas intrahospitalarias, lo cual es afirmado por Celis, et al. (2001) y ratificado por Davis y Newstrom, (1993), quienes aseguran que el entorno sanitario suele proporcionar muchos estímulos generadores de estrés, como lo es el contacto con el paciente, la dinámica de trabajo, la falta de seguridad, entre otras. Abarcando aspectos relacionados con el trabajo en sí y por aspectos derivados de la organización del trabajo Zupiria-Gorostidi (2006). Lo anterior debido principalmente al hecho de tener que desempeñar un nuevo rol para el que aún no se está completamente preparado Ferrer,Rojo, Ruiz, Fernández, San Millán y Martínez (2002). 


\section{CONCLUSIONES}

Según la autopercepción de los estudiantes de enfermería de tercer año en prácticas clínicas intrahospitalarias, del total de la muestra estudiada, se puede concluir que una relevante mayoría se sintió estrés al inicio de estas prácticas. El nivel de estrés se relaciona con la falta de competencia, dentro de la cual destaca como estresor que un alto porcentaje teme "Meter la pata” durante la realización de estas prácticas.

En relación con la sobrecarga que presentan los estudiantes de enfermería en el transcurso de sus prácticas clínicas intrahospitalarias, destaca como altamente preocupante la “sobrecarga de trabajo”. Esto también está relacionado con la sobrecarga académica de los otros módulos de la carrera.

Finalmente, podemos concluir que es importante evaluar el nivel de estrés de los estudiantes, ya que es un factor que puede incidir negativamente en el logro de los aprendizajes. Para ello los docentes deben generar un clima adecuado que facilite los aprendizajes significativos a través de la confianza, el buen trato y la comunicación efectiva.

\section{REFERENCIAS}

Antolín Rodríguez R., Purialto Durán MJ, Moure Fernández ML, Quinteiro Antolín T. (2007) Situaciones de las prácticas clínicas que provocan estrés en los estudiantes de enfermería. Enferm. Glob. file://C:/Users/User/ Downloads/264-Texto\%20del\%20art\%C3\%ADculo-1129-3-10-20080301\%20(1).pdf

Aravena, A. (2017). Principales situaciones que generan estrés en estudiantes de enfermería, durante la práctica clínica, en distintos semestres académicos. Tesis Doctoral http://repositorio.unab.cl/xmlui/handle/ria/5802

Celis, Juan, y Bustamante, Marco, y Cabrera, Dino, y Cabrera, Magno, y Alarcón, Walter, y Monge, Eduardo (2001). Ansiedad y Estrés Académico en Estudiantes de Medicina Humana del Primer y Sexto Año. Anales de la Facultad de Medicina, 62 (1), 25-30.

Cobo Cuenca AI; Carbonell Gómez de Zamora R, Rodríguez Aguilera C, Vivo Ortega I, Castellanos Rainero RM, Sánchez Donaire A. (2010). Estresores y ansiedad de los estudiantes de enfermería en sus primeras prácticas clínicas. NURE Inv. [Revista en Internet]. file://C:/Users/User/Downloads/513-Texto\%20del\%20art\%C3\%ADculo-2031-1-10-20150618\%20(1).pdf

Da Silva, M. (2006). Nuevas perspectivas de la Calidad de Vida Laboral y sus relaciones con la eficacia organizacional. Tesis Doctoral, Universidad de Barcelona, España. http://diposit.ub.edu/dspace/bitstream/2445/42743/1/ MDS_TESIS.pdf

Davis, K., y Newstrom, J. (1993). Comportamiento humano en el trabajo. México: Mc Graw-Hill.Octava edición.

De Dios Duarte, Ma José, Varela Montero, Isabel, Braschi Diaferia, Lorenzo, y Sánchez Muñoz, Esther. (2017). Estrés en estudiantes de enfermería. Educación Médica Superior, 31(3), 110-123. .

Eyssautier M. (2006). Metodología de la investigación. México D.F.: Editorial Thomson.

Ferrer Pascual MaA, Rojo Pascual MaC, Ruiz Gómez MaC, Fernández Araque AM, San Millán MG, y Martínez León, JC.(2002) Análisis situacional de las prácticas clínicas en la Escuela de Enfermería de Soria. Metas Enferm. 5 (44): $18-22$.

Forrellat Barrios, Mariela. (2014). Calidad en los servicios de salud: un reto ineludible. Revista Cubana de Hematología, Inmunología y Hemoterapia, 30(2), 179-183.

Gonzáles, M. C. (2017). Satisfacción Laboral del profesional de Enfermería del Servicio de Emergencias del Hospital "José Casimiro Ulloa” 2015. Tesis de Especialización. Universidad Nacional Mayor de San Marcos. Lima, Perú. Obtenido de:

https://cybertesis.unmsm.edu.pe/bitstream/handle/20.500.12672/7104/Gonzales_mm.pdf?sequence=1\&isAllowed=y Granados, I. (2011). Calidad de Vida Laboral: Historia, Dimensiones y Beneficios. Revista IPS, Facultad de Psicología, Universidad Nacional Mayor de San Marcos, 14(2), 271-276.

Greenglass, E. R., Burke, R. J., \& Fiksenbaum, L. (2001). Workload and burnout in nurses. Journal of Community \& Applied Social Psychology, 11, 211-215.

López González, J.M., Orbañanos Peiro, L., y Cibanal Juan, M.L. (2013). Identificación de estresores en las prácticas clínicas y evolución de los mismos en una promoción de alumnos de enfermería. Sanidad Militar, 69(1), 13-21.

López V, Francisca, y López M., María José. (2011). Situaciones Generadoras de Estrés en los Estudiantes de Enfermería en las Prácticas Clínicas. Ciencia y enfermería, 17 (2), 47-54.

López, I. y Sánchez, V. (2005). Percepción del estrés en estudiantes de enfermería en las prácticas clínicas. Publicado en Enfermería Clínica. vol.15 núm. 06.

McGrath A., Reid N, Boore J.(2003) Occupational stress in nursing. Int J Nurs Stud, 40:555-65.

Mahat G. (1996) Stress and coping: first-year Nepalese nursing students in clinical settings. J Nurs Educ., 35(4):163169.

Moya Nicolás, María, Larrosa Sánchez, Sandra, López Marín, Cristina, López Rodríguez, Irene, Morales Ruiz, Lidia, y Simón Gómez, Ángela. (2013). Percepción del estrés en los estudiantes de enfermería ante sus prácticas clínicas. Enfermería Global, 12(31), 232-253.

Pérez Andrés, Cristina, Alameda Cuesta, Almudena, y Albéniz Lizarraga, Carmen. (2002). La formación práctica en enfermería en la Escuela Universitaria de Enfermería de la Comunidad de Madrid. Opinión de los alumnos y de los profesionales asistenciales: Un estudio cualitativo con grupos de discusión. Revista Española de Salud Pública, $76(5), 517-530$. 
Velasquez A., Cornejo C., Bustamante F., y Mella M. (2018).Estresores que influven desfavorablemente en estudiantes chilenos de Obstetricia y Puericultura, durante las prácticas clínicas. Facultad de Medicina. Universidad de Chile Santiago. EDUMECENTRO vol. 10 n3 versión On-line ISSN 2077-2874

Zupiria Gorostidi, X., Uranga Iturriotz, M.J., Alberdi Erize, M.J., y Barandiaran Lasa, M. (2003). KEZKAK: a new bilingual questionnaire to measure nursing students' stressors in clinical practice. Gaceta Sanitaria, 17(1), 37-50.

Zupiria, X, Uranga, J., Alberdi, J., y otros, (2006). Fuentes de estrés en la práctica clínica de los estudiantes de enfermería. Evolución a lo largo de la diplomatura. Enferm Clin;16(5):231-7. 